\title{
Original
}

\section{Impacto de la contaminación ambiental en los ingresos hospitalarios pediátricos: estudio ecológico}

\author{
Marciano Sánchez Bayle ${ }^{a}$, Raquel Martín Martín ${ }^{b}$, Enrique Villalobos Pinto ${ }^{c}$ \\ apediatra. Fundación para la Investigación, Estudio y Desarrollo de la Salud Pública. Madrid. España \\ - bPediatra. CS Párroco Julio Morate. Madrid. España • 'Servicio de Pediatría. Hospital Niño Jesús. \\ Madrid. España.
}

Objetivo: estudiar la relación existente entre los niveles de contaminantes atmosféricos y los ingresos hospitalarios pediátricos totales y por patología respiratoria en particular.

Pacientes y métodos: estudio ecológico en el cual la variable dependiente analizada han sido los ingresos pediátricos generales y aquellos por patología respiratoria, concretamente neumonías, crisis asmáticas y bronquiolitis, en un hospital del centro de Madrid durante seis años (2012-2017). Como variables independientes se estudiaron los valores promedio de contaminantes ambientales registrados en la ciudad de Madrid. Se calcularon coeficientes de correlación y regresión lineal múltiple. Se comparó el promedio de ingresos cuando los valores de dióxido de nitrógeno $\left(\mathrm{NO}_{2}\right)$ eran superiores e inferiores a $40 \mu \mathrm{g} / \mathrm{m}^{3}$.

Resultados: durante el periodo de tiempo estudiado se registraron 10512 ingresos en Pediatría general, $5328(50,68 \%)$ causados por procesos respiratorios. Se encontró una correlación entre los niveles de $\mathrm{NO}_{2}, \mathrm{CO}$, benceno y los ingresos hospitalarios totales y respiratorios en todos los casos con un valor de $p<0,0001$. En la regresión lineal múltiple los ingresos totales se relacionaron con los niveles de $\mathrm{NO}_{2}$ positiva y negativamente con la temperatura, en relación con los ingresos respiratorios se incrementan con los niveles de $\mathrm{NO}_{2}$ y benceno y disminuyen con la temperatura. Se calculó que si los niveles de $\mathrm{NO}_{2}$ no hubieran superado en ningún mes los niveles de $40 \mu \mathrm{g} / \mathrm{m}^{3}$ se podrían haber evitado el $8,37 \%$ (IC 95 : 7,77 a 8,98) de los ingresos totales y el 6,73\% (IC 95: 6 a 7,52) de los ingresos respiratorios.

Palabras clave:

- Contaminación medioambiental

- Enfermedades respiratorias - Ingresos hospitalarios Conclusiones: se encontró una relación entre los ingresos totales y por enfermedad respiratoria en la infancia y los niveles de contaminantes atmosféricos, especialmente $\mathrm{NO}_{2}$. La mejora de la calidad del aire podría evitar un porcentaje significativo de ingresos pediátricos y propiciarla debería ser tarea prioritaria para los pediatras.

\section{Impact of environmental contamination on pediatric hospital admissions: ecological study}

Objective: to study the relationship between air pollution levels and total pediatric hospital admissions and due to respiratory pathologies in particular.

Patients and methods: ecological study in which the dependent variable analyzed was total pediatric admissions and those due to respiratory pathologies, specifically pneumonias, asthma attacks, bronchiolitis, in a hospital in the centre of Madrid and for 6 years (2012-2017). As independent variables, the average values of environmental pollutants registered in the city of Madrid were studied. Correlation coefficients and multiple linear regression were calculated. The average hospital admissions was compared when the values of nitrogen dioxide $\left(\mathrm{NO}_{2}\right)$ were higher and lower than $40 \mathrm{\mu g} / \mathrm{m}^{3}$.

Results: during the period of the study, there were a total of 10512 admissions in general pediatrics, out of which $5328(50.68 \%)$ caused by respiratory processes. A correlation was found between the levels of $\mathrm{NO}_{2}, \mathrm{CO}$, benzene and the total and respiratory-related hospital admissions in all cases with a value of $p<0.0001$. In the multiple linear regression, the total admissions were related to $\mathrm{NO}_{2}$ levels positively and negatively with the temperature. As for the admissions due to respiratory pathology, they increase with the levels of $\mathrm{NO}_{2}$ and benzene and decrease with temperature. It was calculated that if the levels of $\mathrm{NO}_{2}$ had not exceeded $40 \mu \mathrm{g} / \mathrm{m}^{3}$ in any month, $8.37 \%(95 \mathrm{Cl}: 7.77-8.98)$ of the total admissions and $6.73 \%$ (95 Cl: 6-7.52) of respiratory-related admissions could have been avoided.

Key words: Conclusions: a relation was found between the total pediatric hospital admissions and those due to respiratory pathologies and the levels of air pollutants, especially $\mathrm{NO}_{2}$. The improvement of air quality could prevent a significant percentage of pediatric admissions. It should be a priority for pediatricians to encourage the improvement of air quality.

- Hospital admissions

- Respiratory diseases

Cómo citar este artículo: Sánchez Bayle M, Martín Martín R, Villalobos Pinto E. Impacto de la contaminación ambiental en los ingresos hospitalarios pediátricos: estudio ecológico. Rev Pediatr Aten Primaria. 2019;21:21-9. 


\section{INTRODUCCIÓN}

La salud es un derecho fundamental del ser humano y respirar un aire limpio contribuye a ello en buena medida ${ }^{1}$. La calidad del aire se ve alterada notablemente por la presencia en el mismo de una serie de sustancias de diversa naturaleza que resultan tóxicas para la salud y que conocemos como contaminantes ${ }^{2}$. La contaminación medioambiental supone, por lo tanto, una concentración excesiva en la atmósfera de dióxido de nitrógeno $\left(\mathrm{NO}_{2}\right)$, monóxido de carbono (CO), dióxido de azufre $\left(\mathrm{SO}_{2}\right)$, hidrocarburos como el benceno, y las partículas en suspensión de tamaño inferior a 2,5 $\mu$ y $10 \mu$, entre otros. En las grandes ciudades, como es el caso de Madrid ${ }^{3,4}$, la polución es noticia habitual y la prensa publica imágenes donde la famosa boina refleja la suciedad del aire que respiramos. Organismos internacionales como la Organización Mundial de la Salud (OMS) ${ }^{5}$, la Agencia Europea de Medio Ambiente y organizaciones como Ecologistas en Acción ${ }^{6}$, emiten periódicamente informes con datos obtenidos de manera objetiva e informan y alertan sobre los efectos nocivos que tiene la polución atmosférica para la población en general y para la infancia en particular.

"iNo contamines mi futuro!"” y "Herencia de un mundo sostenible: atlas sobre salud infantil y medio ambiente" ${ }^{8}$ constituyen dos de los últimos informes de la OMS sobre riesgos medioambientales que afectan a la población infantil y en ellos se hace hincapié en la vulnerabilidad de este colectivo. Ciertas características anatomofisiológicas marcadas por la inmadurez de sus sistemas y aparatos, así como unos comportamientos propios de la infancia (los niños más pequeños gatean, se llevan a la boca las manos y objetos variados) y también aspectos sociales en sus hábitos de vida (pasan mucho tiempo al aire libre) son la razón por la cual son menos capaces que los adultos de neutralizar y eliminar de su organismo los contaminantes externos ${ }^{9}$.

Cada vez son más numerosos los estudios que pretenden contribuir a producir un cuerpo creciente de evidencias científicas que en un momento determinado se puedan traducir en políticas de salud pública ${ }^{10}$. Establecer un vínculo causal entre factores ambientales y sus efectos perjudiciales para la salud plantea muchas dificultades y, teniendo en cuenta el elevado coste que supone atender las enfermedades derivadas de la mala calidad del aire que respiramos ${ }^{11}$, el objetivo de nuestro trabajo ha sido analizar la influencia de la contaminación medioambiental en los ingresos hospitalarios pediátricos en general y concretamente los ingresos por procesos respiratorios como las neumonías, las bronquiolitis y las crisis asmáticas. Asimismo, hemos analizado los ingresos que se habrían conseguido evitar en el caso de no haber superado los niveles de $\mathrm{NO}_{2}$ de $40 \mu \mathrm{g} / \mathrm{m}^{3}$, que es el nivel máximo de este contaminante que recomienda la OMS.

\section{PACIENTES Y MÉTODOS}

Se trata de un estudio ecológico, realizado con los datos obtenidos de los informes de alta del Servicio de Pediatría General del Hospital Infantil del Niño Jesús, situado en el centro de Madrid. Se han seleccionado y considerado como la variable dependiente del estudio los ingresos hospitalarios pediátricos en general y concretamente los ingresos producidos por patologías respiratorias como neumonías, bronquiolitis y crisis asmáticas en pacientes de 0 a 18 años de edad y durante el periodo comprendido entre el 1 de enero del 2012 y el 31 de diciembre del 2017 (72 meses).

Los datos referentes a la contaminación medioambiental se han obtenido de la página web del Ayuntamiento de Madrid ${ }^{12}$ (Área de Gobierno de Medio Ambiente y Movilidad), Dirección General de Sostenibilidad y Planificación de la Movilidad, Servicio de Protección de la atmósfera. Los datos empleados en este estudio de los diferentes contaminantes $\left(\mathrm{NO}_{2}, \mathrm{SO}, \mathrm{CO}\right.$, benceno y partículas en suspensión de tamaño inferior a 2,5 $\mu$ y $10 \mu$ ) incluyendo además la temperatura y durante los meses a estudio, han sido los valores promedio mensuales de las 24 estaciones remotas automáticas que recogen la información básica para la vigilancia atmosférica en la ciudad de Madrid. Hemos considerado que de esta forma se valora mejor el contenido 
ambiental al que se ven sometidos los niños que ingresan en el hospital puesto que proceden de todos los ámbitos de la ciudad. Se calculó además el número de ingresos que podrían haberse evitado en el caso de que en ninguno de los meses estudiados se hubieran alcanzado los niveles de $40 \mu \mathrm{g} / \mathrm{m}^{3}$ de $\mathrm{NO}_{2}$.

El análisis estadístico de los datos se realizó aplicando el programa SPSS 15.0. Los datos básicos se expresaron en medias y desviaciones estándar en el caso de las variables cuantitativas y en números y porcentajes en el caso de las variables cualitativas. Se calcularon los intervalos de confianza del 95\% (IC 95).

Las comparaciones entre las variables cuantitativas se realizaron mediante el test de Mann-Whitney después de comprobar que no se ajustaban a una distribución normal (test de Kolmogorov-Smirnov). En las que se realizaron entre las variables cualitativas se utilizó la prueba de $\chi^{2}$. Se consideró significación estadística para p valores inferiores a 0,05.

Se analizaron los coeficientes de correlación de Spearman entre todas las variables cuantitativas estudiadas.

Posteriormente se realizó un análisis multivariante mediante regresión lineal múltiple, partiendo del modelo máximo y retirando de una en una las variables que no tenían significación estadística $(p<0,05)$. Se calculó el número de ingresos evitables si los niveles de $\mathrm{NO}_{2}$ no hubieran superado los $40 \mu \mathrm{g} / \mathrm{m}^{3}$.

\section{RESULTADOS}

En el periodo de tiempo estudiado hubo un total de 10512 ingresos en Pediatría general, 5328 de ellos $(50,68 \%)$ causados por procesos respiratorios. El rango de edad de los pacientes era de 0-18 años, con una edad media de 2,84 años de los pacientes ingresados en general y de 2,38 años en el caso de los pacientes ingresados por patología respiratoria.

En la Tabla 1 se muestra el análisis descriptivo de las variables incluidas en nuestro trabajo. La media de ingresos por mes fue de 146 en los ingresos totales y de 74 en los ingresos respiratorios. Se observa una gran variabilidad entre los valores mensuales del número de ingresos totales y respiratorios y del nivel de los contaminantes (como se observa en la diferencia entre los valores máximo y mínimo de todas estas variables). La Tabla 2 recoge los coeficientes de correlación de Spearman encontrados entre los contaminantes, la temperatura y los ingresos totales y respiratorios. El $\mathrm{NO}_{2}$, el $\mathrm{CO}$, el benceno y la temperatura presentaron coeficientes de correlación con una significación estadística $<0,0001$ (en el último caso negativa) $\mathrm{SO}_{2}$ y partículas materiales con un diámetro <10 $\mu$ (PM 10) también tuvieron correlaciones con significación estadística (en el segundo caso, PM10 negativa) y solo las partículas materiales con un diámetro $<2,5 \mu$ (PM 2,5) no tuvieron correlación con ninguno de los dos tipos de ingresos analizados.

\begin{tabular}{|l|c|c|c|c|}
\hline Tabla 1. Estadísticos descriptivos de las variables incluidas en nuestro estudio \\
\hline & Mínimo & Máximo & Media & Desviación típica \\
\hline Ingresos totales mensuales $(\mathrm{n})$ & 32 & 277 & 142,1667 & 54,25775 \\
\hline Ingresos por patología respiratoria mensuales $(\mathrm{n})$ & 5 & 213 & 72,2833 & 51,37502 \\
\hline Benceno $\left(\mu \mathrm{g} / \mathrm{m}^{3}\right)$ & 0,20 & 1,80 & 0,7617 & 0,42748 \\
\hline $\mathrm{SO}_{2}\left(\mu \mathrm{g} / \mathrm{m}^{3}\right)$ & 1 & 19 & 8,0833 & 4,20771 \\
\hline $\mathrm{CO}\left(\mu \mathrm{g} / \mathrm{m}^{3}\right)$ & 0,10 & 0,70 & 0,4033 & 0,14018 \\
\hline $\mathrm{NO}_{2}\left(\mu \mathrm{g} / \mathrm{m}^{3}\right)$ & 9 & 157 & 43,2667 & 30,63501 \\
\hline $\mathrm{PM} 10\left(\mu \mathrm{g} / \mathrm{m}^{3}\right)$ & 9 & 44 & 23,3725 & 8,13378 \\
\hline $\mathrm{PM} 2,5\left(\mu \mathrm{g} / \mathrm{m}^{3}\right)$ & 7 & 26 & 13,7241 & 4,34799 \\
\hline Temperatura $\left(^{\circ} \mathrm{C}\right)$ & 5,40 & 30 & 15,7968 & 7,28142 \\
\hline
\end{tabular}

CO: monóxido de carbono; $\mathrm{NO}_{2}$ : dióxido de nitrógeno; PM 10: partículas materiales con un diámetro <10 $\mu ;$ PM 2,5: partículas materiales con un diámetro $\left\langle 2,5 \mu ; \mathrm{SO}_{2}\right.$ : dióxido de azufre. 


\begin{tabular}{|c|c|c|}
\hline & $\begin{array}{l}\text { Ingresos } \\
\text { totales }\end{array}$ & $\begin{array}{l}\text { Ingresos } \\
\text { respiratorios }\end{array}$ \\
\hline $\mathrm{NO}_{2}$ & $\begin{array}{c}0,564 \\
p<0,0001\end{array}$ & $\begin{array}{c}0,597 \\
p<0,0001\end{array}$ \\
\hline PM 10 & $\begin{array}{c}-0,391 \\
p=0,005\end{array}$ & $\begin{array}{c}0,277 \\
p=0,049\end{array}$ \\
\hline PM 25 & $\begin{array}{c}-0,146 \\
p=0,274\end{array}$ & $\begin{array}{c}-0,014 \\
p=0,915\end{array}$ \\
\hline $\mathrm{CO}$ & $\begin{array}{c}0,669 \\
p<0,0001\end{array}$ & $\begin{array}{c}0,648 \\
p<0,0001\end{array}$ \\
\hline $\mathrm{SO}_{2}$ & $\begin{array}{c}0,378 \\
p=0,003\end{array}$ & $\begin{array}{c}0,443 \\
p<0,0001\end{array}$ \\
\hline Benceno & $\begin{array}{c}0,633 \\
p<0,0001\end{array}$ & $\begin{array}{c}0,672 \\
p<0,0001\end{array}$ \\
\hline Temperatura $\left({ }^{\circ} \mathrm{C}\right)$ & $\begin{array}{c}-0,813 \\
p<0,0001\end{array}$ & $\begin{array}{c}-0,732 \\
p<0,0001\end{array}$ \\
\hline
\end{tabular}

$\mathrm{CO}$ : monóxido de carbono; $\mathrm{NO}_{2}$ : dióxido de nitrógeno; $\mathrm{PM} \mathrm{10:}$ partículas materiales con un diámetro <10 $\mu$; PM 2,5: partículas materiales con un diámetro $\left\langle 2,5 \mu ; \mathrm{SO}_{2}\right.$ : dióxido de azufre.

Se constató que los meses en los que los niveles de $\mathrm{NO}_{2}$ eran igual o superiores a $40 \mu / \mathrm{m}^{3}$ el porcentaje de ingresos fue mayor de manera significativa que el de los meses en que no se alcanzaron estos niveles.

Se realizaron estudios de regresión lineal múltiple tanto para los ingresos totales como para los respiratorios (Tablas 3 y 4) en las que se observa que para los ingresos totales presentaron significación estadística los valores de $\mathrm{NO}_{2}$ (asociación positiva) y la temperatura (negativa). En el caso de los ingresos respiratorios se mantuvieron en el modelo los valores de $\mathrm{NO}_{2}$ y el benceno (valores positivos) y la temperatura (negativos).

Las Figs. 1 y 2 recogen las curvas característica operativa del receptor (COR) de los valores obtenidos en la regresión lineal múltiple para los ingresos totales $A B C=0,884$ (IC 95: 0,795 a 0,973; $p<0,0001$ ) y los ingresos por patología respiratoria por encima de la media, $A B C=0,929$ (IC 95: 0,864 a 0,994; $p<0,0001)$.

La cifra de ingresos totales evitables si el $\mathrm{NO}_{2}$ hubiera sido inferior a $40 \mu \mathrm{g} / \mathrm{m}^{3}$ fue de 880 y de ellos $360(40,90 \%)$ por procesos respiratorios (Tabla 5). Finalmente se calculó el porcentaje de ingresos evitables si los valores de $\mathrm{NO}_{2}$ hubieran sido inferiores a $40 \mu \mathrm{g} / \mathrm{m}$ durante todo el periodo de tiempo estudiado, que fueron el $8,37 \%$ de los ingresos totales (IC 95: 7,77 a 8,98) y el 6,73\% (IC 95: 6-7 a 52 ) de los ingresos respiratorios.

\section{DISCUSIÓN}

En 2005 la OMS estableció unos valores límites recomendados para cada uno de los contaminantes, con la advertencia de que la presencia de dichas sustancias por encima de dichos valores supone un grave riesgo para el equilibrio medioambiental $^{13}$, entendiendo por medioambiente el espacio donde se desarrolla la vida y que incluye el aire, el agua, el suelo, los seres vivos y las relaciones que se crean entre ellos. La contaminación es responsable

Tabla 3. Resultados de la regresión lineal múltiple para ingresos totales

\begin{tabular}{|l|c|c|c|}
\hline & Coeficiente $\beta$ & Intervalo de confianza del 95\% & $p$ \\
\hline $\mathrm{NO}_{2}$ & 1,113 & 0,385 a 1,841 & 0,003 \\
\hline Temperatura & $-5,151$ & $-6,354 \mathrm{a}-3,949$ & 0,0001 \\
\hline
\end{tabular}

$\mathrm{NO}_{2}$ : dióxido de nitrógeno.

$\mathrm{R} 2=0,709, p<0,0001$.

Tabla 4. Resultados de la regresión lineal múltiple para ingresos respiratorios

\begin{tabular}{|l|c|c|c|}
\hline & Coeficiente $\beta$ & Intervalo de confianza del 95\% & $p$ \\
\hline $\mathrm{NO}_{2}$ & 0,411 & 0,025 a 0,797 & 0,037 \\
\hline Benceno & 31,041 & 2,187 a 59,896 & 0,035 \\
\hline Temperatura & $-3,178$ & $-4,639$ a $-1,718$ & $<0,0001$ \\
\hline
\end{tabular}

$\mathrm{NO}_{2}$ : dióxido de nitrógeno.

$\mathrm{R} 2=0,651, p<0,0001$. 


\begin{tabular}{|l|c|c|c|c|c|}
\hline \multicolumn{6}{|c|}{ Tabla 5. Comparación entre los ingresos pediátricos totales y respiratorios según valores de $\mathrm{NO}_{2}$ de $\geq 40 \mu \mathrm{g} / \mathrm{m}^{3}$} \\
\hline & Nivel $\left(\mu \mathrm{g} / \mathrm{m}^{3}\right)$ & $\mathrm{n}$ & Media & Desviación típica & $p$ \\
\hline Ingresos totales & $\geq 40$ & 52 & 147,7609 & 57,41319 & 0,011 \\
& $<40$ & 20 & 123,7857 & 38,41910 & \\
\hline Ingresos respiratorios & $\geq 40$ & 52 & 78,8261 & 55,64822 & 0,010 \\
& $<40$ & 20 & 50,7857 & 24,70185 & \\
\hline
\end{tabular}

de casi dos millones de muertes infantiles cada año en el mundo y más de 30000 en Europa, según la $\mathrm{OMS}^{14}$.

Dada la importancia del tema, desde hace años se vienen desarrollando por parte de organismos internacionales, gobiernos, grupos de investigación una serie de estrategias y proyectos centrados en factores ambientales concretos y relacionados con la infancia, que es la población más vulnerable ${ }^{15,16}$. De especial interés en nuestro país es el proyecto Infancia y Medio Ambiente (INMA) ${ }^{17}$ que se inicia en 1998. Consiste en una red con varias líneas de investigación abiertas y está dirigido por Jordi Sunyer et al. Comenzó estudiando a las madres gestantes de 4000 niños y hace un seguimiento del

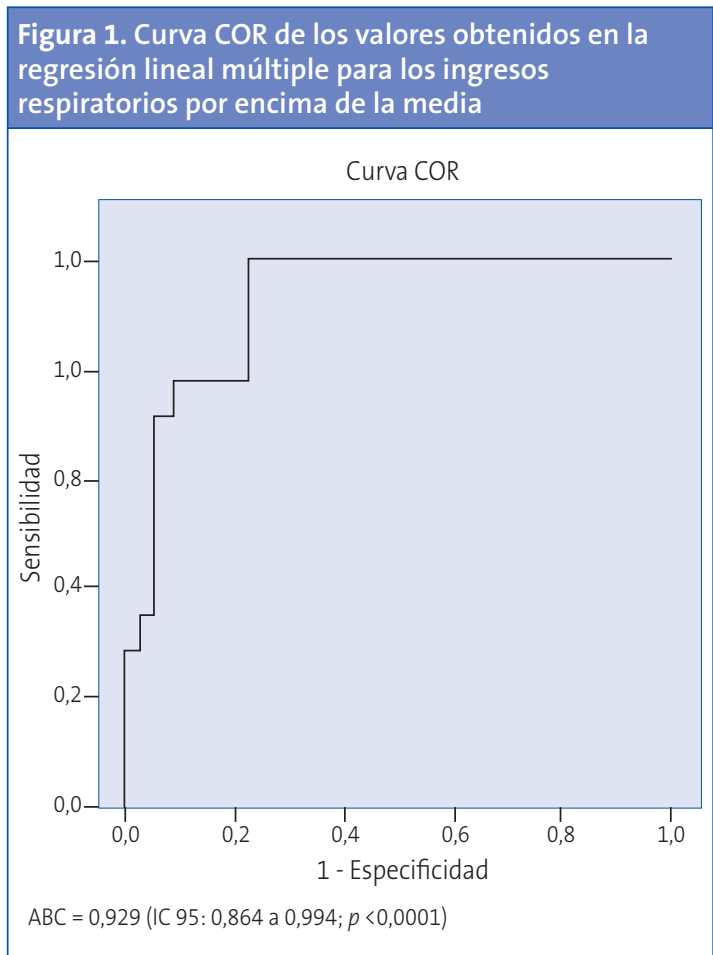

papel de los contaminantes las patologías que presentaban hasta los 18 años relacionándolas con los niveles de polución atmosférica. Las conclusiones de todos los proyectos y estudios realizados es que el desarrollo físico, social e intelectual del niño requiere un ambiente protegido y protector, por lo cual la contaminación constituye el mayor riesgo para la salud infantil.

La bibliografía consultada sobre el tema suele hacer referencia a la relación existente entre las enfermedades del aparato respiratorio infantil ${ }^{18-20}$, por ser las más frecuentes, especialmente las crisis asmáticas y la contaminación atmosférica, de tal forma que los ingresos hospitalarios por esta causa aumentan notablemente coincidiendo con los

Figura 2. Curva COR de los valores obtenidos en la regresión lineal múltiple para los ingresos totales por encima de la media

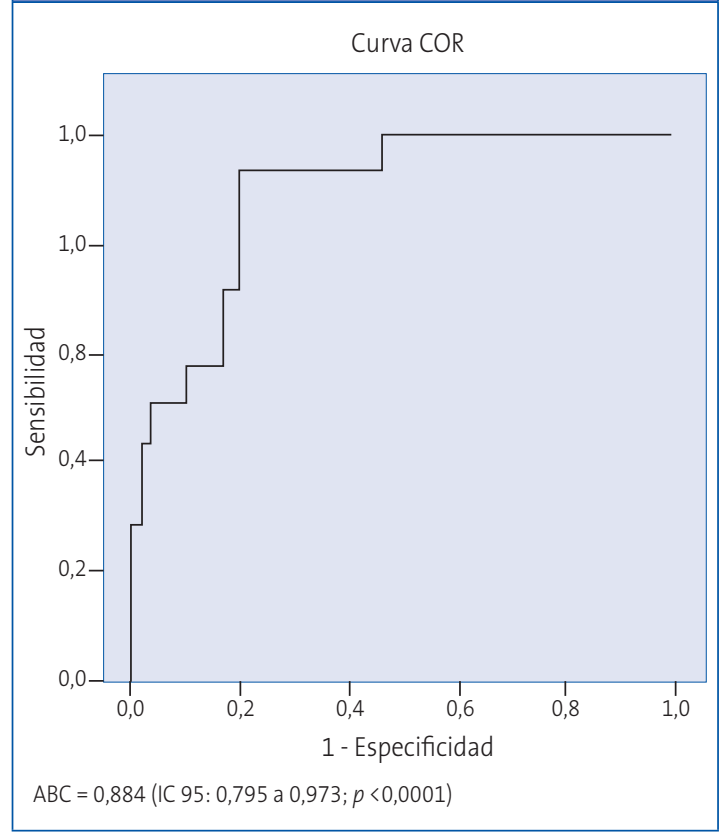


picos de contaminación ${ }^{21-22}$; nuestros resultados coinciden en ese sentido. Nuestro estudio muestra también un incremento de los ingresos totales, es decir, que incluimos los ingresos por otras causas que se ven incrementados cuando los niveles de $\mathrm{CO}$, benceno y $\mathrm{NO}_{2}$ se elevan. Negrisoli et al. ${ }^{23}$ han encontrado un aumento de los ingresos por neumonías en concreto. Otras investigaciones asocian la contaminación atmosférica con otro tipo de patologías, Kaplan et al. ${ }^{24}$ relacionan algunos casos de apendicitis con la exposición al ambiente contaminado. Son numerosos los estudios que alertan sobre la influencia en la capacidad de atención en los niños que se vería disminuida en los días en que están expuestos a mayores índices de polución ${ }^{25-27}$. Mortamais et al. ${ }^{28}$ han encontrado relación con el déficit de atención y la hiperactividad. El trabajo de Yang et al. ${ }^{29}$ relaciona el aire contaminado con el autismo.

El informe emitido por la organización Ecologistas en Acción sobre la calidad del aire en España durante $2016^{30}$ pone de manifiesto que las administraciones no actúan con la suficiente contundencia, por eso en 2015 la Comisión Europea inició un procedimiento de infracción contra España por el incumplimiento de la normativa sobre la calidad del aire respecto al $\mathrm{NO}_{2}$, contaminante que procede sobre todo de las emisiones de los vehículos.

Los resultados de nuestro estudio confirman los niveles excesivos de $\mathrm{NO}_{2}$, CO y benceno registrados en las estaciones de medición situadas en la ciudad. Estos resultados son similares a los encontrados por Martín Rivada et al. ${ }^{31}$ en su trabajo. La mayoría de la bibliografía seleccionada sobre efectos de la polución en la infancia son estudios que se han realizado en hospitales y suponen, por lo tanto, los casos de pacientes con mayor grado de afectación.

Se han señalado recientemente los resultados obtenidos en las consultas de Atención Primaria ${ }^{32}$ por patología respiratoria y los valores de contaminantes, la patología ambulatoria pone de manifiesto los casos menos graves y por tanto los más habituales. Independientemente del ámbito donde se realice el estudio, se ha encontrado una relación significativa entre ingresos totales e ingresos respiratorios, además de las consultas ambulatorias, con los elevados niveles de contaminantes, especialmente el $\mathrm{NO}_{2}$ por encima de los $40 \mathrm{~g} / \mathrm{m}^{3}$. En los últimos años, las estaciones de la capital ofrecen datos que rebasan los límites recomendados por la Unión Europea (UE) que, por otro lado, son incluso más permisivos que los valores máximos recomendados por la OMS y, por lo tanto, más protectores. Las ciudades españolas por lo general sobrepasan los niveles de $\mathrm{NO}_{2}$ deseables y el estudio de Linares et al. ${ }^{33}$ muestra cómo está influyendo a corto plazo en las cifras de mortalidad de la población. En España y en el espacio de tiempo comprendido entre los años 2000 y 2009, una reciente investigación de Díaz et al. ${ }^{34}$ relaciona la concentración de ozono atmosférico con el incremento de la mortalidad por patología cardiorrespiratoria.

El efecto negativo que tiene la temperatura en la regresión lineal de nuestro trabajo es posible que esté relacionado con el hecho de que las bajas temperaturas favorecen la propagación de ciertos virus y por lo tanto con las infecciones respiratorias, que son la causa más frecuente de ingreso en la infancia ${ }^{35}$

El informe emitido por el Ministerio ${ }^{36}$ respecto al $\mathrm{NO}_{2}$ en el Estado español en 2016 presenta una mejora respecto al año anterior, sin embargo, y concretamente en las grandes ciudades como Madrid, se superan los valores protectores que recomienda la OMS para diferentes sustancias contaminantes.

Se trata de unir esfuerzos, aumentando la colaboración entre gobiernos, organizaciones no gubernamentales y organismos internacionales, así como la aportación de los estudios llevados a cabo por profesionales de la salud.

El índice de desempeño ambiental, Environmental Performance Index ${ }^{37}$, con 20 años de experiencia, sitúa en 2018 a España en el puesto 12 de 180 países. Este informe es emitido cada dos años por la Universidad de Yale y evalúa las políticas de conservación de ecosistemas y desempeño ambiental de un país. 
Las Unidades de Salud Medioambiental Pediátrica en Europa ${ }^{38-39}$, creadas en EE. UU. en 1998 son unidades clínicas donde profesionales, incluidos pediatras, evalúan y reconocen la influencia de factores medioambientales en la patología de los pacientes, con lo cual ratifican la posición privilegiada del pediatra en el ámbito de la salud medioambiental con el fin de proteger a la infancia.

Nuestro estudio tiene las limitaciones propias de los estudios ecológicos; la fundamental es que toman a la población como unidad de estudio, es decir, que observamos la asociación entre una exposición y sus resultados en un grupo de población, sin embargo, no es posible relacionar de manera individual la exposición de un individuo con su estado de salud. Como novedad, introducimos los datos obtenidos a lo largo de estos años sobre los ingresos evitables tanto generales como por patología respiratoria.

En conclusión, los resultados de nuestro estudio ratifican que existe una relación entre los ingresos pediátricos y los niveles de contaminación atmosférica en la ciudad, de tal manera que los picos de contaminación aumentan los ingresos hospitalarios generales y respiratorios y que dichos ingresos disminuyen cuando los niveles de $\mathrm{NO}_{2}$ en concreto

\section{BIBLIOGRAFÍA}

1. Gascón M, Sunyer J. Contaminación del aire y salud respiratoria en niños. Arch Bronconeumol. 2015;5: 371-2.

2. Oyarzún M. Contaminación aérea y sus efectos en la salud. Rev Chil Enf Resp. 2010;26:16-25.

3. Caballero F. La contaminación aumenta en 2017 en Madrid e incumple por octavo año consecutivo la normativa europea. En: Eldiario.es [en línea] [consultado el 11/01/2019]. Disponible en www.eldiario.es/ contaminación-Madrid-incumple-consecutivo-nor mativa_0_730027237.html

4. Cerrillo A. La población urbana expuesta a aire sucio es, según la OMS, 8 veces superior a lo que dicen los datos de la UE. En: La Vanguardia [en línea] [consultado el descienden. Además, nuestro trabajo pone de manifiesto que controlar la polución evita en muchos casos ingresos hospitalarios. La contaminación es perjudicial incluso a niveles bajos y a corto plazo, sin que exista un umbral sin efectos adversos para la salud de las personas ${ }^{40}$. Teniendo en cuenta que la fuente principal de $\mathrm{NO}_{2}$ es el tráfico rodado, en las grandes ciudades se impone controlar y reducir este, aportando medidas que favorezcan otras opciones de movilidad ciudadana. La relación entre salud y medio ambiente debería de considerarse como parte esencial de las decisiones estratégicas en materia de política económica y de desarrollo global.

\section{CONFLICTO DE INTERESES}

Los autores declaran no presentar conflictos de intereses en relación con la preparación y publicación de este artículo.

\section{ABREVIATURAS}

CO: monóxido de carbono - COR: característica operativa del receptor • IC 95: intervalo de confianza del 95\% • $\mathbf{N O}_{2}$ : dióxido de nitrógeno - OMS: Organización Mundial de la Salud - PM 10: partículas materiales con un diámetro <10 $\mu$ •PM 2,5: partículas materiales con un diámetro $<2,5 \mu \cdot \mathrm{SO}_{2}$ : dióxido de azufre.

11/01/2019]. Disponible en www.lavanguardia.com/ natural/20161124/412124325578/polucion-habi tantes-ciudades-oms-ue.html

5. Calidad del aire ambiente (exterior) y salud. En: Organización Mundial de la Salud [en línea] [consultado el 11/01/2019]. Disponible en www.who.int/es/ news-room/fact-sheets/detail/ambient-(outdoor)air-quality-and-health

6. La calidad del aire en la ciudad de Madrid, 2017. En: Ecologistas en Acción [en línea] [consultado el 11/01/2019]. Disponible en www.ecologistasenac cion.org/IMG/pdf/info-calidad-aire-madrid-2017.pdf

7. ¡No contamines mi futuro! El impacto de los factores medioambientales en la salud infantil. En: Organización Mundial de la Salud [en línea] [consultado el 11/01/2019]. Disponible en http://apps.who.int/iris/ handle/10665/260430 
8. ¿La herencia de un mundo sostenible? Atlas sobre salud infantil y medio ambiente. Ginebra: Organización Mundial de la Salud; 2018.

9. Linares C, Díaz J. Efectos de las partículas de diámetro inferior a 2,5 micras PM $(2,5)$ sobre los ingresos hospitalarios en niños menores de 10 años en Madrid. Gac Sanit. 2009;23:192-7.

10. Sunyer J, Ribas-Fito N. Efectos ambientales en la salud infantil. En: El País [en línea] [consultado el 11/01/2019]. Disponible en https://elpais.com/dia rio/2003/04/08/salud/1049752802-850215.html

11. Informe del Lancet Countdown sobre salud y cambio climático, 2017. El inicio de una transformación a nivel mundial para la salud pública después de 25 años de pasividad. En: The Lancet [en línea] [consultado el 11/01/2019]. Disponible en www.thelancet.com/ pb/assets/raw/Lancet/Hubs/climate-change/Lan cet_Countdown_2017_Executive_Summary_Spa nish.pdf

12. Sistema Integral de la Calidad del Aire, 2016. En: Ayuntamiento de Madrid [en línea] [consultado el 11/01/2019]. Disponible en www.mambiente.muni madrid.es/sica/scripts/index.php.

13. Air quality guidelines for particulate matter, ozone, nitrógeno dioxide and sulfur dioxide. Global update 2005. En: Organización Mundial de la Salud [en línea] [consultado el 11/01/2019]. Disponible en http://apps.who.int/iris/bitstream/handle/10665/ 69477/WHO_SDE_PHE_OEH_06.02_eng.pdf;jsessi onid $=46$ BF13BC6C7FF027BFBB8424A8F7D2BE? seq uence $=1$.

14. Las consecuencias de la contaminación ambiental: 1,7 millones de defunciones infantiles anuales, según la OMS, 6 de marzo de 2017. En: Organización Mundial de la Salud [en línea] [consultado el 11/01/2019]. Disponible en www.who.int/es/newsroom/detail/06-03-2017-the-cost-of-a-polluted-en vironment-1-7-million-child-deaths-a-year-sayswho

15. The Lancet. Turning climate change legislation into public health policy. Lancet. 2018;391:1865.

16. Sandín Vázquez M, Sarriá Santamera A. Evaluación de impacto en salud y medio ambiente. Agencia de Evaluación de Tecnologías Sanitarias (AETS). Madrid: Instituto de Salud Carlos III; 2007.

17. Ramón R, Ballester F, Rebagliato M, Ribas N, Torrent M, Fernández $M$, et al. La red de investigación "Infancia y medioambiente" (Red INMA): protocolo de estudio. Rev Esp Salud Pública. 2005;79:203-20.

18. Gavidia T, Pronczuk J, Sly PD. Impactos ambientales sobre la salud respiratoria de los niños. Carga global de las enfermedades respiratorias pediátricas ligada al ambiente. Rev Chil Enf Resp. 2009;25:99-108.

19. Vargas S, Onatra W, Osorno L, Páez E, Sáenz O. Contaminación atmosférica y efectos respiratorios en niños, en mujeres embarazadas y en adultos mayores. Revista UDCA. Actualidad y Divulgación Científica. 2008;11:31-45.

20. Salazar-Ceballos A, Álvarez-Miño L. Los efectos del material particulado 10 (PM 10) y de las variables climatológicas en las admisiones hospitalarias por enfermedades respiratorias en niños de la ciudad de Santa Marta, Colombia, 2008-2009. Revista Facultad de Ciencias de la Salud. 2011;8:127-42.

21. Haneen J, Nieuwenhuijsen MJ. Traffic-related air poIlution and chilhood asthma: recent advances and remaining gaps in the exposure assessment methods. Int Environ Res Public Health. 2017;14:312.

22. Elizalde-Beiras I, Guillén-Grima F, Aguinaga-Ontoso I. Factores asociados al asma en los niños y adolescentes en la zona rural de Navarra (España). Aten Primaria. 2018;50:332-9.

23. Negrisoli J, Fernando L, Nascimento C. Atmospheric pollutants and hospital admissions due to pneumonia in children. Rev Paul Pediatr. 2013;31:501-6.

24. Kaplan GG, Dixon E, Panaccione R, Fong A, Chen L, Szyszkowicz $M$, et al. Effect of ambient air pollution on the incidence of apendicitis. CMAJ. 2009;181:591-7.

25. Costa LG, Cole TB, Coburn J, Chang Y, Dao K, Roqué PJ. Neurotoxicity of traffic-related air pollution. Neurotoxicology. 2017;59:133-9.

26. Sunyer J, Esnaola M, Álvarez-Pedrerol M, Forns J, Rivas I, López-Vicente M, et al. Association between trafficrelated air pollution in schools and cognitive development in primary school children: a prospective cohort study. PLoS Med. 2015;12:e1001792.

27. Suades-González E, Gascón M, Guxens M, Sunyer J. Air pollution and neuropsychological development: a review of the latest evidence. Endocrinology. 2015; 156:3473-82.

28. Mortamais M, Pujol J, van Drooge BL, Macia D, Martínez- Vilavella G, Reynes C, et al. Effect of exposure to polycyclic aromatic hydrocarbons on basal ganglia and attention-deficit hyperactivity disorder symptoms in primary school children. Environ Int. 2017;105:12-9. 
29. Yang C, Zhao W, Deng K, Zhou V, Zhou X, Hou Y. The association between air pollutants and autism spectrum disorders. Environ Sci Pollut Resp Int. 2017;24: 15949-58.

30. La calidad del aire en el Estado español durante 2017. En: Ecologistas en Acción [en línea] [consultado el 11/01/2019]. Disponible en www.ecologistase naccion.org/wp-content/uploads/2018/06/infor me-calidad-aire-2017.pdf

31. Martín Rivada A, Sánchez Bayle M, Villalobos Pinto E, Calleja Gero ML. Contaminación ambiental e ingresos pediátricos en un área urbana. Acta Pediatr Esp. 2018;76:44-9.

32. Martín Martín R, Sánchez Bayle M. Impacto de la contaminación ambiental en las consultas pediátricas de Atención Primaria: estudio ecológico. An Pediatr (Barc). 2017;89:80-5.

33. Linares C, Falcón I, Ortiz C, Díaz J. An approach estimating the short-term effect of $\mathrm{NO}_{2}$ on daily mortality in Spanish cities. Environ Int. 2018;116:18-28.

34. Díaz J, Ortiz C, Falcón I, Salvador C, Linares C. Shortterm effect of tropospheric ozone on daily mortality in Spain. Atmospheric Environ. 2018;187:107-16.
35. García García ML, Korta Murua J, Callejón A. Bronquiolitis aguda viral. Protoc Diagn Ter Pediatr. 2017; 1:85-102.

36. Evaluación de la calidad del aire en España 2016. En: Ministerio de Agricultura y Pesca, Alimentación y Medio Ambiente [en línea] [consultado el 11/01/ 2019]. Disponible en www.mapama.gob.es

37. Hsu A, Zomer A. Environmental Performance Index. En: Wiley Stats Ref. Statistics Reference Online [en línea] [consultado el 11/01/2019]. Disponible en https://onlinelibrary.wiley.com/action/showCitForm ats?doi=10.1002\%2F9781118445112.stat03789. pub2

38. Ortega García JA, Ferris i Tortajada J, Claudio Morales L, Berbel Tornero O. Unidades de salud medioambiental pediátrica en Europa: de la teoría a la acción. An Pediatr (Barc). 2005;63:143-51.

39. Campillo I, López F, Ortega-García JA. Pediatría ambiental: la salud de los niños y el medio ambiente. Pediatr Integral. 2018;22:155e1-155e6.

40. Di Q, Dai L, Wang Y. Association of short-term exposure to air pollution with mortality in older adults. JAMA. 2017;318:2446-56. 